\title{
On the Remainder in Quadrature Rules
}

\author{
By P. D. Tuan
}

\begin{abstract}
An expression is obtained for the remainder in quadrature rules applied to functions whose Hilbert transforms exist. The estimation of the remainder is illustrated by means of a particular example.
\end{abstract}

1. Introduction. Let $w(t)$ be a given function, nonnegative in a real interval $[a, b]$ and integrable over $(a, b)$. Suppose that to approximate the integral

$$
\int_{a}^{b} w(t) f(t) d t
$$

we use a quadrature rule of order $n$ (see, for example, [1]). That is, we replace the integral by a weighted sum

$$
\sum_{r=0}^{n} \lambda_{r, n} f\left(t_{r, n}\right)
$$

where the numbers $\lambda_{r, n}$ are called the weight factors and $t_{r, n}$, the nodes of the rule, are $n$ distinct points in $[a, b]$. The free parameters $\lambda_{r, n}$ and $t_{r, n}$ may be chosen in different manners which result in a variety of quadrature rules. In particular, we are interested in quadrature rules which are exact, i.e. $R_{n}[f]=0$, when $f(t)$ is a polynomial of degree less than $n$. It may be shown [1] that for such rules,

$$
\lambda_{r, n}=-\psi_{n}\left(t_{r, n}\right) / \phi_{n}^{\prime}\left(t_{r, n}\right),
$$

where $\phi_{n}(t)$ is a polynomial of degree $n$ with zeros at $t=t_{r, n}, r=1,2, \cdots, n$, and $\psi_{n}\left(t_{r, n}\right)$ is given by

$$
\psi_{n}\left(t_{r, n}\right)=\int_{a}^{b} \frac{w(t) \phi_{n}(t)}{t_{r, n}-t} d t .
$$

The remainder $R_{n}[f]$ in a quadrature rule is defined by

$$
R_{n}[f]=\int_{a}^{b} w(t) f(t) d t-\sum_{r=0}^{n} \lambda_{r, n} f\left(t_{r, n}\right) .
$$

For the particular rules mentioned above, the remainder may be expressed as a contour integral (see, for example, [2]),

$$
R_{n}[f]=\frac{1}{2 \pi i} \int_{\mathrm{e}} \frac{\psi_{n}(z)}{\phi_{n}(z)} f(z) d z
$$

where

Received December 7, 1970.

AMS 1970 subject classifications. Primary 65D30, 41A55, 44A15.

Key words and phrases. Remainder, quadrature rules, Hilbert transform, Cauchy principal value, contour integral. 


$$
\psi_{n}(z)=\int_{a}^{b} \frac{w(t) \phi_{n}(t)}{z-t} d t
$$

The interval $[a, b]$ is contained in the interior of the contour $\mathfrak{C}$ on and within which the function $f(z)$ is analytic.

In the application of quadrature rules arise the questions of convergence and rate of convergence of the sum (1.2) to the integral (1.1). Both questions are answered if either the remainder can be expressed explicitly as a function of $n$ or its asymptotic behaviour is known for large values of $n$. Starting from the contour integral form (1.6), asymptotic estimates for the remainder have been obtained in [2] [5]. It is interesting to note that these asymptotic estimates, although derived for large $n$, often are surprisingly close to the actual values of the remainder for relatively small $n$. It is our aim in this paper to derive an expression, and hence obtain estimates, for the remainder in quadrature rules applied to a special class of functions. The functions which we shall consider are those $f(t)$ for which the Hilbert transforms

$$
F(x)=\frac{1}{\pi} f_{-\infty}^{\infty} \frac{f(t)}{t-x} d t \quad(x \text { real })
$$

exist, $f_{-\infty}^{\infty}$ denoting the Cauchy principal value of $\int_{-\infty}^{\infty}$.

2. An Expression for the Remainder. We shall give our principal result in Theorem 1, but first we need two lemmas.

LEMMA 1 [6, THEOREM 101]. If a function $f(t)$ belongs to the class $L_{p}, p>1$, in the basic interval $(-\infty, \infty)$, then (1.8) defines almost everywhere a function $F(x)$, which also belongs to $L_{p}$, whose Hilbert transform coincides almost everywhere with $-f(t)$.

LEMMA 2. Let $f(t)$ and $g(t)$ be functions of the classes $L_{p}$ and $L_{a}$, respectively. If $1 / p+1 / q<1$, then

$$
f_{-\infty}^{\infty} \frac{f(t)}{t-t_{0}} d t f_{-\infty}^{\infty} \frac{g(x)}{x-t} d x=f_{-\infty}^{\infty} g(x) d x f_{-\infty}^{\infty} \frac{f(t) d t}{\left(t-t_{0}\right)(x-t)}-\pi^{2} f\left(t_{0}\right) g\left(t_{0}\right) .
$$

Proof. This lemma follows from a result in [7, p. 171] to which the reader is referred for details.

We may now state and prove the theorem.

THEOREM 1. Suppose that

(i) $w(t)$ is a nonnegative function in $[a, b]$ and integrable over $(a, b) ; w_{1}(t)$ is the function defined by

$$
\begin{aligned}
w_{1}(t) & =w(t), & & (a \leqq t \leqq b), \\
& =0, & & (t<a, t>b) .
\end{aligned}
$$

(ii) $\phi_{n}(t)$ is a polynomial of degree $n$ with simple zeros at $t=t_{r, n}$, where $a \leqq t_{r, n} \leqq b$ or $r=1,2, \cdots, n$.

(iii) $w_{1}(t) \phi_{n}(t)$ is in $L_{a}(-\infty, \infty), q>1$, and the Hilbert transform $\Phi_{n}(x)$ of $w_{1}(t) \phi_{n}(t)$ exists.

(iv) $f(t)$ is in $L_{p}(-\infty, \infty), p>1$, and the Hilbert transform $F(x)$ of $f(t)$ exists.

(v) $p$ and $q$ are such that $1 / p+1 / q<1$.

Then the remainder in the quadrature rule defined by 


$$
R_{n}[f]=\int_{a}^{b} w(t) f(t) d t-\pi \sum_{r=1}^{n} \frac{\Phi_{n}\left(t_{r, n}\right)}{\phi_{n}^{\prime}\left(t_{r, n}\right)} f\left(t_{r, n}\right)
$$

may be expressed as

$$
R_{n}[f]=f_{-\infty}^{\infty} \frac{\Phi_{n}(x)}{\phi_{n}(x)} F(x) d x .
$$

Proof. We shall prove the theorem starting from (2.4). From condition (iii) and the definition of the Hilbert transformation (1.8), we may write (2.4) as

$$
R_{n}[f]=f_{-\infty}^{\infty} \frac{F(x)}{\phi_{n}(x)} d x\left\{\frac{1}{\pi} f_{-\infty}^{\infty} \frac{w_{1}(t) \phi_{n}(t)}{t-x} d t\right\} .
$$

Since $\phi_{n}(t)$ has $n$ simple zeros at $t=t_{r, n}, r=1,2, \cdots, n$, we may use partial fractions to obtain

$$
\frac{1}{\phi_{n}(t)}=\sum_{r=1}^{n} \frac{1}{\phi^{\prime}\left(t_{r, n}\right)\left(t-t_{r, n}\right)}
$$

Substituting (2.6) into (2.5), we get

$$
R_{n}[f]=\sum_{r=1}^{n} \frac{1}{\phi^{\prime}\left(t_{r, n}\right)} f_{-\infty}^{\infty} \frac{F(x)}{x-t_{r, n}} d x\left\{\frac{1}{\pi} f_{-\infty}^{\infty} \frac{w_{1}(t) \phi_{n}(t)}{t-x} d t\right\} .
$$

From condition (iv) and Lemma $1, F(x)$ belongs to $L_{p}$; furthermore, $w_{1}(t) \phi_{n}(t)$ is in $L_{a}$ by condition (iii), $p$ and $q$ being related as in condition (v). Thus, we may apply Lemma 2 to the iterated integral in (2.7) to get

$$
R_{n}[f]=\sum_{r=1}^{n} \frac{1}{\phi^{\prime}\left(t_{r, n}\right)} f_{-\infty}^{\infty} w_{1}(t) \phi_{n}(t) d t\left\{\frac{1}{\pi} f_{-\infty}^{\infty} \frac{F(x) d x}{\left(x-t_{r, n}\right)(t-x)}\right\}
$$

The constant term vanishes as $t_{r, n}$ are the zeros of $\phi_{n}(t)$. Writing

$$
\frac{1}{\left(x-t_{r, n}\right)(t-x)}=\frac{1}{t-t_{r, n}}\left(\frac{1}{x-t_{r, n}}-\frac{1}{x-t}\right) \text {, }
$$

and making use of Lemma 1 , we deduce that

$$
\frac{1}{\pi} f_{-\infty}^{\infty} \frac{F(x) d x}{\left(x-t_{r, n}\right)(t-x)}=\frac{f(t)-f\left(t_{r, n}\right)}{t-t_{r, n}}
$$

The Eqs. (2.8) and (2.9) give, after some rearrangement,

$$
\begin{aligned}
R_{n}[f]= & f_{-\infty}^{\infty} w_{1}(t) \phi_{n}(t) f(t)\left[\sum_{r=1}^{n} \frac{1}{\phi_{n}^{\prime}\left(t_{r, n}\right)\left(t-t_{r, n}\right)}\right] d t \\
& -\sum_{r=1}^{n} \frac{f\left(t_{r, n}\right)}{\phi_{n}^{\prime}\left(t_{r, n}\right)} f_{-\infty}^{\infty} \frac{w_{1}(t) \phi_{n}(t)}{t-t_{r, n}} d t,
\end{aligned}
$$

which, upon using (2.2), (2.6) and the definition of $\Phi_{n}(x)$, becomes

$$
R_{n}[f]=\int_{a}^{b} w(t) f(t) d t-\pi \sum_{r=1}^{n} \frac{\Phi_{n}\left(t_{r, n}\right)}{\phi_{n}^{\prime}\left(t_{r, n}\right)} f\left(t_{r, n}\right),
$$

and the theorem is proved. 
The expression (2.4) bears a great resemblance to the contour integral form (1.6). In addition, the Hilbert transform

$$
\Phi_{n}(x)=\frac{1}{\pi} f_{-\infty}^{\infty} \frac{w_{1}(t) \phi_{n}(t)}{t-x} d t \equiv \frac{1}{\pi} f_{a}^{b} \frac{w(t) \phi_{n}(t)}{t-x} d t
$$

is connected to the function $\psi_{n}(z)$, defined by (1.7), through the relation

$$
\begin{aligned}
\Phi_{n}(x) & =-\frac{1}{2 \pi}\left[\psi_{n}(x+i 0)+\psi_{n}(x-i 0)\right], & & (a \leqq x \leqq b), \\
& =-\frac{1}{\pi} \psi_{n}(x), & & (x<a, x>b) .
\end{aligned}
$$

For $x=t_{r, n}, r=1,2, \cdots, n$, we have

$$
\Phi_{n}\left(t_{r, n}\right)=-\frac{1}{\pi} \psi_{n}\left(t_{r, n}\right),
$$

since the function $\psi_{n}(z)$ is uniquely defined at these points. Thus, the quadrature rules defined by (2.3) are identical to those given in the previous section.

Theorem 1 applies in particular to Gaussian quadrature rules. The more familiar ones are those in which $\phi_{n}(t)$ is a classical orthogonal polynomial:

$$
\begin{array}{lllll}
\text { Quadrature rule } & (a, b) & \phi_{n}(t) & w(t) \\
\text { Gauss-Jacobi } & (-1,1) & P_{n}^{(\alpha, \beta)}(t) & (1-t)^{\alpha}(1+t)^{\beta} \quad(\alpha, \beta>-1) \\
\text { Gauss-Laguerre } & (0, \infty) & L_{n}^{(\alpha)}(t) & t^{\alpha} e^{-t} \quad(\alpha>-1) \\
\text { Gauss-Hermite } & (-\infty, \infty) & H_{n}(t) & e^{-t,}
\end{array}
$$

The corresponding associated functions $\psi_{n}(z)$ are given respectively by, see [8],

$$
\begin{aligned}
\Pi_{n}^{(\alpha, \beta)}(z)= & \frac{2^{n+\alpha+\beta+1} \Gamma(n+\alpha+1) \Gamma(n+\beta+1)}{\Gamma(2 n+\alpha+\beta+2)(z-1)^{n+1}} \\
& \times_{2} F_{1}\left(n+1, n+\alpha+1 ; 2 n+\alpha+\beta+2 ; \frac{2}{1-z}\right),
\end{aligned}
$$

where ${ }_{2} F_{1}(k, l ; m ; z)$ denotes a hypergeometric function;

$$
\lambda_{n}^{(\alpha)}(z)=-\Gamma(n+\alpha+1) U\left(n+1 ; 1-\alpha ; e^{i \pi} z\right),
$$

where $U(k ; l ; z)$ denotes a confluent hypergeometric function, see [9, p. 5, Eq. (1.3.1)];

$$
\eta_{n}(z)=e^{\mp((n+1) \pi) / 2} \pi^{1 / 2} \Gamma(n+1) U\left(\frac{1}{2}(n+1) ; \frac{1}{2} ; e^{\mp i \pi} z^{2}\right),
$$

where the upper (lower) sign corresponds to $\operatorname{Im} z>0(\operatorname{Im} z<0)$. The functions $\Phi_{n}(x)$ may be deduced from the last three equations, making use of the relation (2.10). In the next section, we shall illustrate, by means of an example, the estimation of the remainder as given in Theorem 1.

3. The Estimation of the Remainder. Consider, for example, the remainder in the Gauss-Jacobi quadrature rule applied to the function 


$$
f(t)=1 /\left(t^{2}+k^{2}\right) \quad(k>0) .
$$

For the Gauss-Jacobi rule, the function $\Phi_{n}(x)$ is defined by $\Pi_{n}^{*(\alpha, \beta)}(x)$ say,

$$
\begin{aligned}
\Pi_{n}^{*(\alpha, \beta)}(x) & \left.=-\frac{1}{2 \pi} \Pi_{n}^{(\alpha, \beta)}(x+i 0)+\Pi_{n}^{(\alpha, \beta)}(x-i 0)\right], & & (-1 \leqq x \leqq 1), \\
& =-\frac{1}{\pi} \Pi_{n}^{(\alpha, \beta)}(x), & & (x<-1, x>1),
\end{aligned}
$$

where $\Pi_{n}^{(\alpha, \beta)}(x)$ is given by $(2.12)$.

From [10, p. 245, Eq. (10)], the Hilbert transform of $f(t)$ is

$$
F(x)=-x / k\left(x^{2}+k^{2}\right) \text {. }
$$

We also have that $1 /\left(t^{2}+k^{2}\right) \in L_{p}$ for any $p>1$, and that for $\alpha, \beta>-1$, $(1-t)^{\alpha}(1+t)^{\beta} P_{n}^{(\alpha, \beta)}(t) \in L_{1+}$, i.e., it belongs to $L_{a}$, where $1 \leqq q \leqq 1+\epsilon, \epsilon$ being a positive constant. From Theorem 1, (3.2), and (3.3) we may write

$$
R_{n}\left[\frac{1}{t^{2}+k^{2}}\right]=-\frac{1}{k} f_{-\infty}^{\infty} \frac{\Pi_{n}^{*(\alpha, \beta)}(x)}{P_{n}^{(\alpha, \beta)}(x)} \cdot \frac{x}{x^{2}+k^{2}} d x
$$

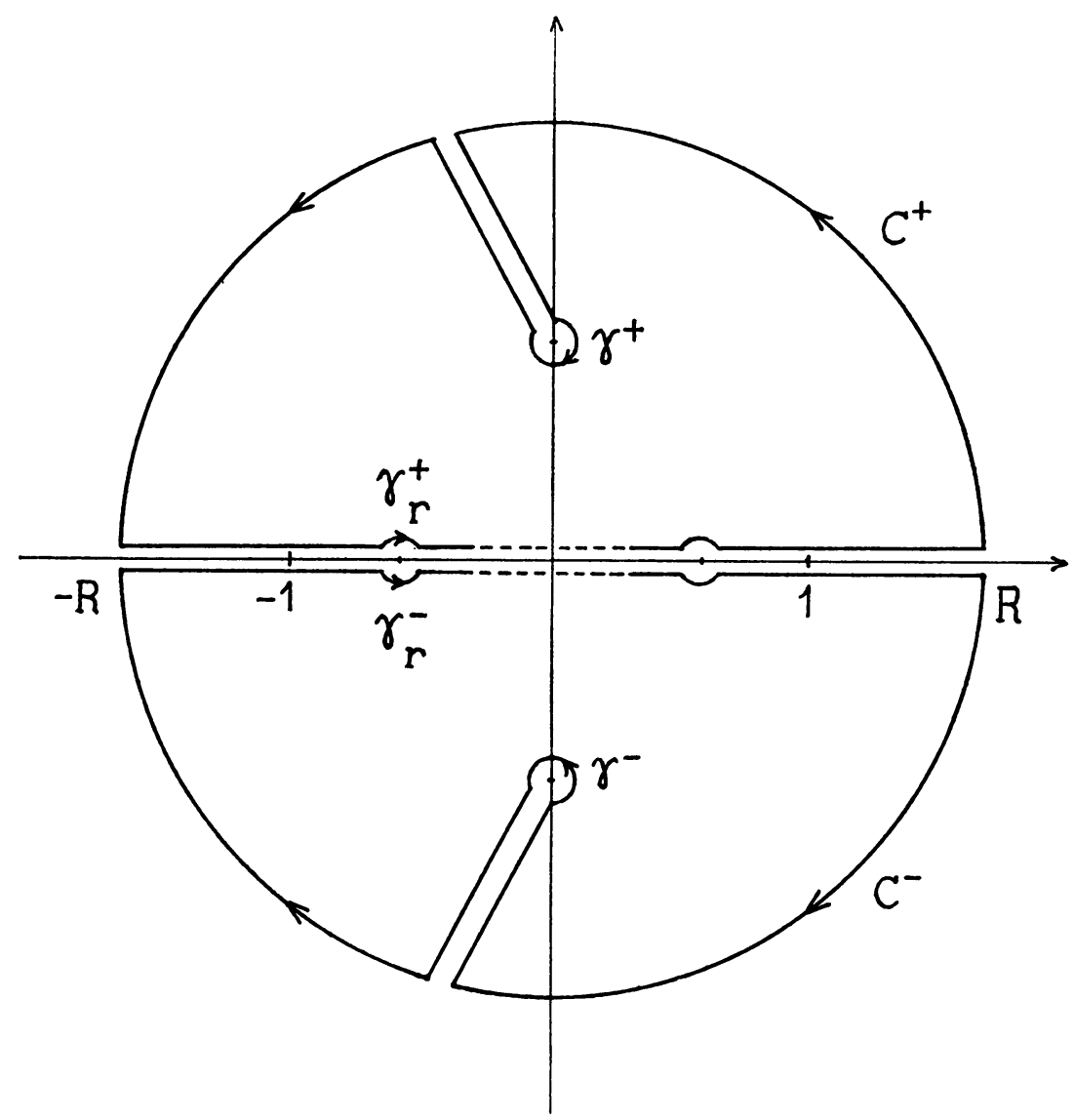

FIGURE 1 
To evaluate the integral in (3.4), let us consider the contour integrals

$$
\left\{\int_{\mathfrak{e}^{+}}+\int_{\mathfrak{e}^{-}}\right\} \frac{\Pi_{n}^{(\alpha, \beta)}(z)}{P_{n}^{(\alpha, \beta)}(z)} \cdot \frac{z}{z^{2}+k^{2}} d z .
$$

The closed contour $\mathfrak{e}^{+}\left(\mathfrak{e}^{-}\right)$which is described in the anticlockwise (clockwise) direction in the upper (lower) half of the complex $z$-plane consists of (see Fig. 1)

(i) a large semicircle $C^{+}\left(C^{-}\right)$with centre at the origin and radius $R$, where $R>1$ and $R>k$, in the upper (lower) half of the $z$-plane,

(ii) a small circle $\gamma^{+}\left(\gamma^{-}\right)$with centre at $z=i k(z=-i k)$ and radius $\rho$,

(iii) a pair of parallel lines joining $C^{+}$and $\gamma^{+}\left(C^{-}\right.$and $\left.\gamma^{-}\right)$,

(iv) a line segment $[-R+i 0, R+i 0]([-R-i 0, R-i 0])$ indented at the zeros $z=t_{r, n}, r=1,2, \cdots, n$, of $P_{n}^{(\alpha, \beta)}(z)$; the indentations are small semicircles $\gamma_{r}^{+}\left(\gamma_{r}^{-}\right)$ with centre at $z=t_{r, n}$ and radius $\rho_{r}$, in the upper (lower) half of the $z$-plane.

It may be shown that

(a) the integrals around $C^{+}$and $C^{-}$tend to zero in the limit as $R \rightarrow \infty$,

(b) the contributions from the semicircles $\gamma_{r}^{+}$and $\gamma_{r}^{-}, r=1,2, \cdots, n$, cancel out,

(c) the net contribution from the parallel lines vanishes.

Furthermore, since the integrand is analytic on and within each of $\mathrm{e}^{+}$and $\mathrm{e}^{-}$, we have

$$
\left\{\int_{\mathfrak{e}^{+}}+\int_{\mathfrak{e}^{-}}\right\} \frac{\Pi_{n}^{(\alpha, \beta)}(z)}{P_{n}^{(\alpha, \beta)}(z)} \cdot \frac{z}{z^{2}+k^{2}} d z=0 .
$$

Thus, letting $R \rightarrow \infty$, we obtain

$$
f_{-\infty}^{\infty} \frac{\Pi_{n}^{*(\alpha, \beta)}(x)}{P_{n}^{(\alpha, \beta)}(x)} \cdot \frac{x}{x^{2}+k^{2}} d x=\frac{1}{2 \pi}\left\{\int_{\gamma}+\int_{\gamma^{-}}\right\} \frac{\Pi_{n}^{(\alpha, \beta)}(z)}{P_{n}^{(\alpha, \beta)}(z)} \cdot \frac{z}{z^{2}+k^{2}} d z .
$$

We further get, from the theory of residues,

$$
\left\{\int_{\gamma^{+}}+\int_{\gamma^{-}}\right\} \frac{\Pi_{n}^{(\alpha, \beta)}(z)}{P_{n}^{(\alpha, \beta)}(z)} \cdot \frac{z}{z^{2}+k^{2}} d z=\pi i\left[\frac{\Pi_{n}^{(\alpha, \beta)}(-i k)}{P_{n}^{(\alpha, \beta)}(-i k)}-\frac{\Pi_{n}^{(\alpha, \beta)}(i k)}{P_{n}^{(\alpha, \beta)}(i k)}\right] .
$$

Finally, from (3.4), (3.6), and (3.7), we deduce that

$$
R_{n}\left[\frac{1}{t^{2}+k^{2}}\right]=\frac{i}{2 k}\left[\frac{\Pi_{n}^{(\alpha, \beta)}(i k)}{P_{n}^{(\alpha, \beta)}(i k)}-\frac{\Pi_{n}^{(\alpha, \beta)}(-i k)}{P_{n}^{(\alpha, \beta)}(-i k)}\right] .
$$

Let us take for example a particular case in which $k=1$ and $a=\beta=0$ (GaussLegendre quadrature rule). Then the remainder is given exactly by

$$
R_{n}\left[\frac{1}{t^{2}+1}\right]=i \frac{\Pi_{n}(i)}{P_{n}(i)},
$$

where $\Pi_{n}(z)=\Pi_{n}^{(0,0)}(z)$ and $P_{n}(z)$ is the Legendre polynomial. Here we have made use of the relations $P_{n}(-z)=(-1)^{n} P_{n}(z)$ and $\Pi_{n}(-z)=(-1)^{n+1} \Pi_{n}(z)$.

From the asymptotic formulae for $\Pi_{n}^{(\alpha, \beta)}(z)$ and $P_{n}^{(\alpha, \beta)}(z)$, see [8], we find that, for large $n$, 


$$
R_{n}\left[\frac{1}{t^{2}+1}\right] \sim \frac{2 \pi(-1)^{n}}{(1+\sqrt{ } 2)^{2 n+1}}
$$

Table 1 shows that the estimated values of the remainder as given by (3.10) compare favourably with the actual values even for small values of $n$.

\begin{tabular}{|c|c|c|}
\hline$n$ & Actual Value & Estimated Value \\
\hline 2 & +0.070796 & +0.076613 \\
\hline 3 & -0.012537 & -0.013144 \\
\hline 4 & +0.002168 & +0.002255 \\
\hline 5 & -0.000375 & -0.000387 \\
\hline 6 & +0.000066 & +0.000066 \\
\hline
\end{tabular}

4. Conclusion. In this paper we have derived an expression for the remainder in quadrature rules applied to the functions whose Hilbert transforms exist. The estimation of the remainder in one particular case has been achieved through examining the related contour integrals. Although only one example was given, the type of analysis which we have employed may in fact be applied to other functions satisfying Theorem 1. It goes without saying that the contours in the related contour integrals must be chosen according to the form of the Hilbert transform of the function under consideration.

Acknowledgments. The author wishes to thank Professor D. Elliott of the University of Tasmania for his valuable guidance. This work was done while the author was in receipt of a University of Tasmania Research Scholarship.

Mathematics Department

University of Western Australia

Nedlands, Western Australia, Australia

Current address:

Mathematics Department

University of Tasmania

Hobart, Tasmania, Australia

1. G. Szegö, Orthogonal Polynomials, 2nd rev. ed., Amer. Math. Soc. Colloq. Publ., vol. 23, Amer. Math. Soc., Providence, R.I., 1959. MR 21 \# 5029.

2. W. BARRETT, "Convergence properties of Gaussian quadrature formulae," Comput. J., v. 3, 1960/61, pp. 272-277. MR 23 \#B1117.

3. W. BARRETT, "On the convergence of Cotes' quadrature formulae," J. London Math. Soc., v. 39, 1964, pp. 296-302. MR 32 \#3272.

4. J. D. Donaldson \& D. ElliotT, Quadrature I: A Unified Approach to the Development of Quadrature Rules, Math. Dept. Technical Report \#23, University of Tasmania, 1970. 
5. J. D. Donaldson \& D. ElliotT, Quadrature II: The Estimation of Remainders in Certain Quadrature Rules, Math. Dept. Technical Report \#24, University of Tasmania, 1970.

6. E. C. Titchmarsh, Introduction to the Theory of Fourier Integrals, Clarendon Press, Oxford, 1937.

7. F. G. Tricomi, Integral Equations, Interscience, New York, 1957. MR 20 \#1177.

8. D. Ellotr, Uniform Asymptotic Expansions of the Classical Orthogonal Polynomials and some Associated Functions, Math. Dept. Technical Report \#21, University of Tasmania, 1970.

9. L. J. Slater, Confluent Hypergeometric Functions, Cambridge Univ. Press, New York, 1960. MR 21 \#5753.

10. A. ERDÉlYI, ET AL., Tables of Integral Transforms, vol. 2, McGraw-Hill, New York, 1954. MR 16, 468. 